\title{
Há alterações dentoesqueléticas no tratamento a longo prazo da apneia obstrutiva do sono com aparelho para avanço mandibular?
}

There are dento-skeletal changes in long-term treatment of obstructive sleep apnea with mandibular advancement
appliance?

KAWAUCHI, Márcia Yuri ' ${ }^{1}$ DAINESI, Eduardo Alvares ${ }^{2}$

1. Doutora em Ortodontia e Ortopedia. Professora do Curso de Odontologia da UNIFIO - Centro Universitário das Faculdades Integradas de Ourinhos, Ourinhos, SP, Brasil.

2. Pós-Doutor em Ortodontia e Ortopedia Facial. Coordenador dos Cursos de Especialização em Ortodontia pela E.Orto-Pós-Graduação em Odontologia, Bauru, SP, Brasil.

Endereço para correspondência:

Márcia Yuri Kawauchi

Rua Luso Brasileira, 4-44 - Sala 1010

Jardim Estoril IV

17016-230 - Bauru - São Paulo - Brasil

E-mail: m.y.k@terra.com.br

Recebido: 30.07 .2019

Aceito: 13.09.2019

\section{RESUMO}

A síndrome da apneia obstrutiva do sono caracteriza-se por repetidos episódios de parada momentânea da respiração, durante o sono, resultando em severas alterações sistêmicas. Há diferentes abordagens para a terapia desta síndrome, dependentes da severidade da mesma. Nas de grau suave ou moderada pode-se indicar uma terapia com aparelhos para avanços mandibulares, associados ou não às máscaras de bombeamento positivo e contínuo de ar nas vias aéreas. Há relatos na literatura indicando o surgimento de mordida cruzada na região dos pré-molares e diminuição do overjet e do overbite, devido à lingualização dos incisivos superiores e vestibularização dos incisivos inferiores, principalmente se o período de utilização do aparelho para avanço mandibular for maior que 3 anos. Este trabalho relata as alterações dentoesqueléticas promovidas pela utilização durante 5 anos de um aparelho intrabucal para avanço mandibular, em um paciente adulto, com quadro de ronco noturno. Pelas análises cefalométricas realizadas ao início do tratamento e após o tempo apresentado, observa-se que as alterações ocorridas não apresentaram relevância clínica. Assim, pode-se esperar que a utilização de aparelhos para avanço mandibular no tratamento da apneia obstrutiva do sono não promove alterações dentoesqueléticas significativas.

Palavras-chave: Apneia obstrutiva do sono. Avanço mandibular. Ortodontia.

\section{ABSTRACT}

The obstructive sleep apnea syndrome is a sleep-related breathing disorder characterized by repeated narrowing or collapse of the upper airway during sleep, resulting in severe systemic alterations. There are different approaches to the therapy, depending on the severity of this syndrome. In mild or moderate levels, therapy with mandibular advancement devices, associated or not with continuous positive airway pressure, may be indicated. There are reports in the literature indicating the occurrence of cross-bite in the premolar region and overjet and overbite reductions, due to the lingualization of the upper incisors and vestibularization of the lower incisors. It occur especially if the period of use of the device for mandibular advancement is greater than 3 years. This study reports the dento-skeletal changes promoted by the use during 5 years of an intra-oral appliance for mandibular advancement in an adult patient with nocturnal snoring. For the cephalometric analyzes performed at the beginning of treatment and after the time presented, it is observed that the changes occurred are not clinically relevant. Thus, it can be expected that the use of mandibular advancement devices in the treatment of obstructive sleep apnea does not promote significant dento-skeletal changes.

Keywords: Sleep apnea, obstructive. Mandibular advancement. Orthodontics. 


\section{INTRODUÇÃO}

A síndrome da apneia obstrutiva do sono (SAOS) caracteriza-se por uma alteração do sono com repetidas interrupções da respiração ${ }^{1-2}$, devido ao estreitamento ou fechamento das vias aéreas superiores. Esta síndrome pode ser classificada como suave, moderada ou severa, de acordo com o número de interrupções respiratórias que ocorrem por hora de sono (índice de deficiência respiratória - IDR), sendo considerada leve se houver de 5 a 14 eventos, moderada se houver de 15 a 29 eventos, e severa se houver acima de 30 eventos por hora ${ }^{3.4}$.

Os aparelhos para avanço mandibular (AAM) parecem ser uma boa indicação na terapia da SAOS suave ${ }^{5-15}$, podendo também ser indicados em casos moderados, quando associados aos aparelhos de bombeamento positivo e contínuo de ar nas vias aéreas (CPAP) ${ }^{5}$. Trata-se de uma opção menos invasiva, de ótima aceitação por parte dos pacientes e de custo relativamente baixo em comparação a outros tipos de abordagens. Os AAM atuam promovendo a protrusão da mandíbula, induzindo a alterações na posição anterior da língua, palato mole e parede lateral da faringe, permitindo assim, uma melhora do fluxo aéreo respiratório ${ }^{6-11,1415}$.

Há relatos na literatura pertinente, que a utilização contínua dos AAM, no tratamento da SAOS, pode causar alterações dentárias como mordida cruzada na região dos pré-molares e diminuição do overjet e do overbite ${ }^{2,12,16-17}$, devido à lingualização dos incisivos superiores e vestibularização dos incisivos inferiores. Estas alterações podem surgir principalmente se o período de utilização dos AAM for maior que $3 \operatorname{anos}^{12,16-17}$ e continuam a ocorrer enquanto o aparelho for utilizado ${ }^{12}$.

Deste modo, este artigo clínico relata as alterações dentoesqueléticas promovidas após a utilização de um aparelho para avanço mandibular, por 5 anos, em um paciente adulto com SAOS.

\section{RELATO DE CASO}

Paciente do gênero masculino, com 26 anos de idade, na época da consulta inicial, foi encaminhado por uma colega otorrinolaringologista, para avaliação ortodôntica e que considerasse a possibilidade de melhora do quadro de roncos durante o sono e da dificuldade para dormir apresentada pelo mesmo. Durante a anamnese, o paciente relatou também certa dificuldade em despertar e xerostomia matinal. Informou ainda que não estava fazendo uso de quaisquer medicamentos e que apresentava uma boa saúde geral, sem históricos de hipertensão ou outra doença relacionada à síndrome da apneia obstrutiva do sono. O paciente trouxe consigo um exame polissonográfico realizado previamente, apresentado os seguintes resultados: redução da eficiência do sono (84.3\%); saturação de oxihemoglobina abaixo de $90 \%$; presença de ronco moderado e índice de deficiência respiratória (IDR) de 3.7, indicando ser um quadro de SAOS leve. Clinicamente apresentava uma má oclusão de Classe I, com severa sobremordida e suave apinhamento anteroinferior (Figura 1). A radiografia panorâmica (ortopantomografia) mostrou um bom paralelismo radicular de quase todos os dentes presentes (Figura 2) e pela telerradiografia em norma lateral, pode-se verificar um bom espaço das vias aéreas posteriores. (Figura 3). O terceiro molar inferior esquerdo apresentava-se não irrompido e com uma angulação mesial muito acentuada, o qual o paciente recusou-se a remover cirurgicamente naquele momento.
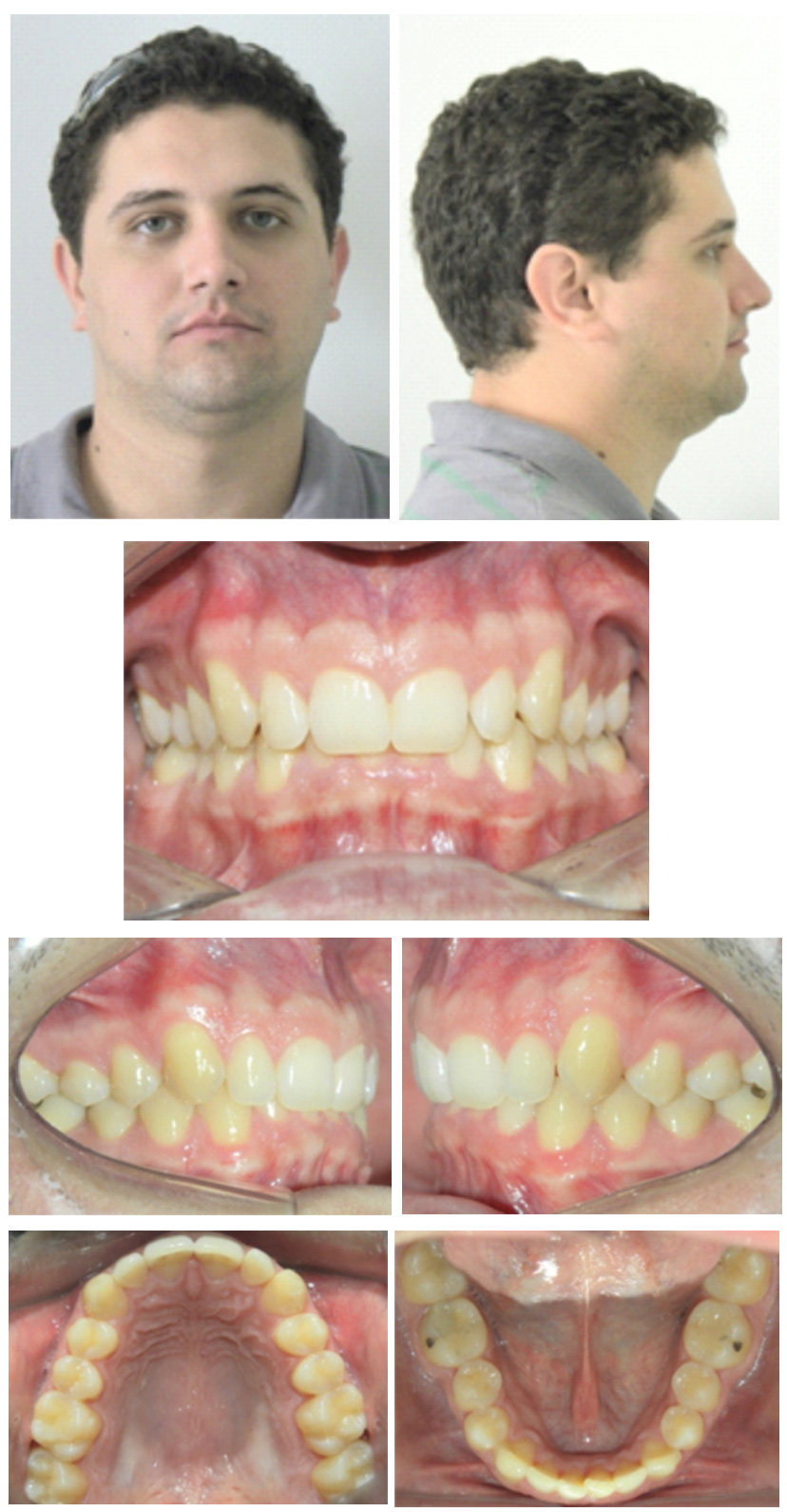

Figura 1 - Fotografias intrabucais iniciais. 


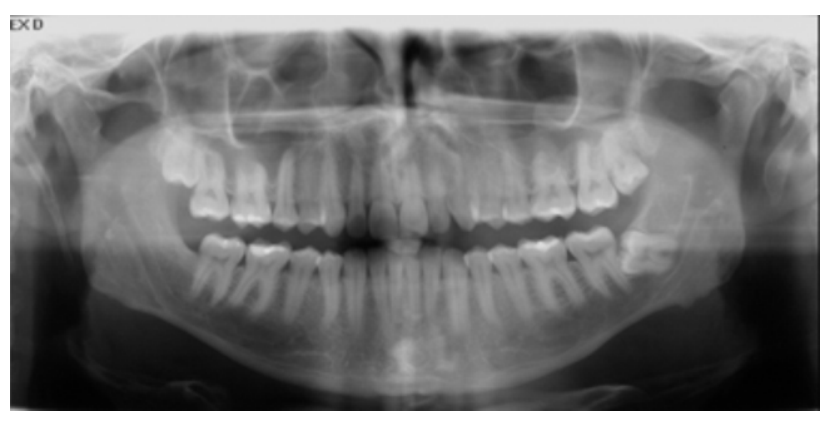

Figura 2 - Radiografia panorâmica inicial.

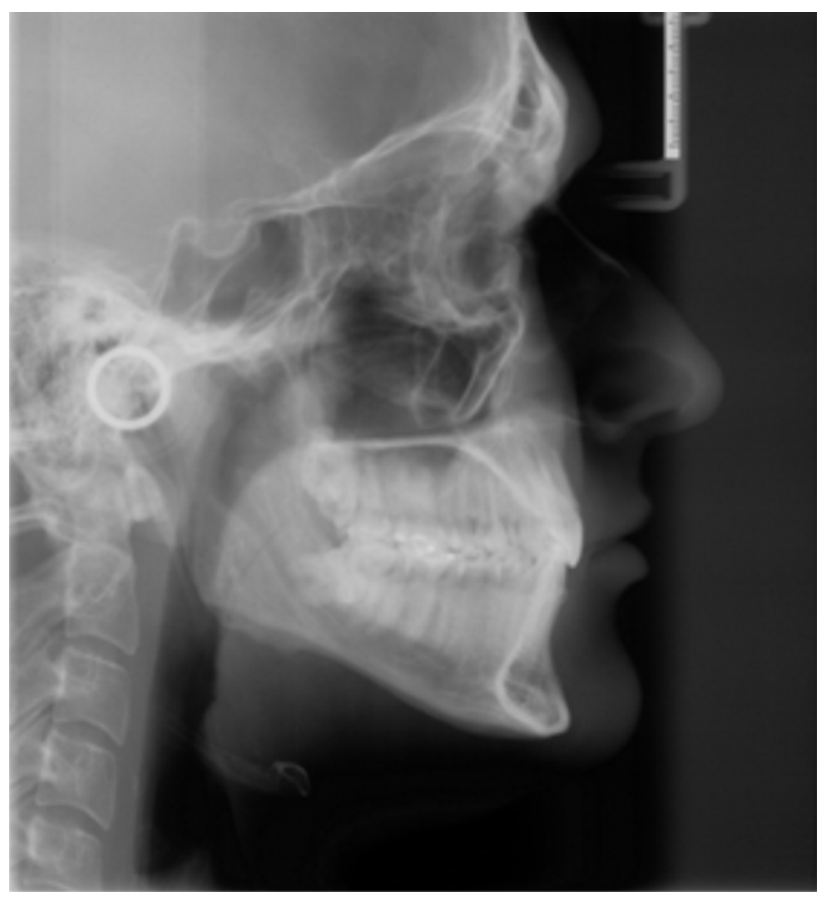

Figura 3 - Telerradiografia em norma lateral inicial.

\section{Abordagem Terapêutica}

Por se tratar de uma SAOS leve e por apresentar uma má oclusão de Classe I com sobremordida acentuada, preconizou-se inicialmente uma abordagem ortodôntica corretiva fixa para correção, principalmente da sobremordida e também do apinhamento anteroinferior, associada a utilização noturna de elásticos de Classe II, para promover um pouco de avanço mandibular e assim, melhorar o quadro presente de ronco. Contudo, o paciente solicitou que a correção da má oclusão fosse postergada e que se sentiria melhor se fosse solucionada prioritariamente a situação de ronco durante o sono. Sendo assim, planejou-se então uma abordagem com aparelho removível para avanço mandibular (AAM $)^{18}$.

\section{Descrição do Aparelho}

Elegeu-se o aparelho de $\mathrm{ITO}^{18}$ para a realização do avanço mandibular. Este aparelho consiste de duas bases de armação metálica fundida (superior e inferior), tipo prótese removível, com resina acrílica termopolimerizável e alças tipo ganchos posicionadas na região ântero-superior e póstero-inferior para posterior utilização de elásticos interarcos de Classe II, com a vantagem de permitir movimentos de lateralidade mandibular, conferindo maior conforto ao paciente ${ }^{15,18}$. O AAM foi confeccionado com registro de mordida em cera com suave avanço mandibular, ou seja, pequeno posicionamento anterior da mandíbula para permitir maior espaço aéreo posterior. Os cuidados com o aparelho incluem guardá-lo em ambiente saturado com água, quando não estiver em uso; e sua higienização deve ser realizada diariamente, antes de sua instalação e após o seu uso, com pasta dentifrícia e escova de dentes e com pastilhas efervescentes, duas ou três vezes por semana. Após dois ou três dias de adaptação, há conforto e comodidade. O paciente foi orientado a renovar os elásticos a cada 4 dias, sendo definido de acordo com a quantidade de força aplicada, somente para manter uma suave protrusão mandibular (Figura 4). Controles subsequentes foram realizados e após um ano de terapia, uma nova telerradiografia lateral foi tomada, com o aparelho em posição (Figura 5). Nesta ocasião também foi realizada uma nova polissonografia. Este novo exame foi feito com o AAM em boca. Os resultados indicaram uma melhora na eficiência do sono (93\%), tornando-se normal; o nível de saturação de oxihemoglobina também se normalizou (90\%), o IDR diminuiu para 2.9, e o mais relevante, não havia mais a presença de roncos noturnos. Os resultados obtidos nos dois exames polissonográficos apresentam-se na Tabela 1.
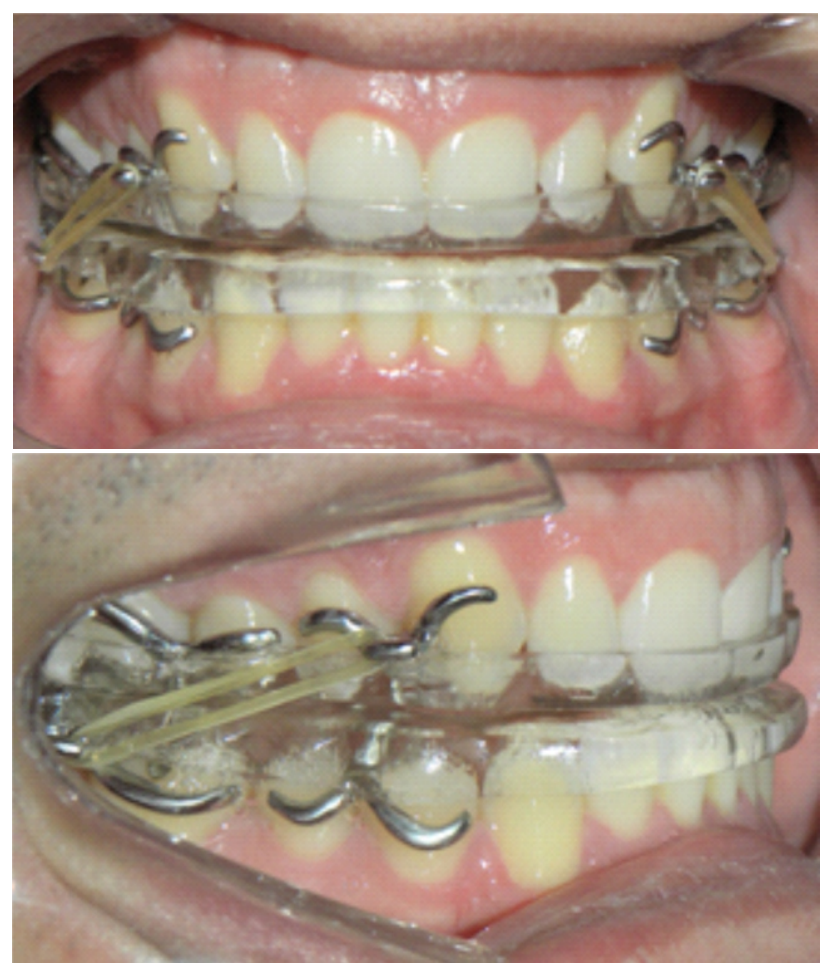


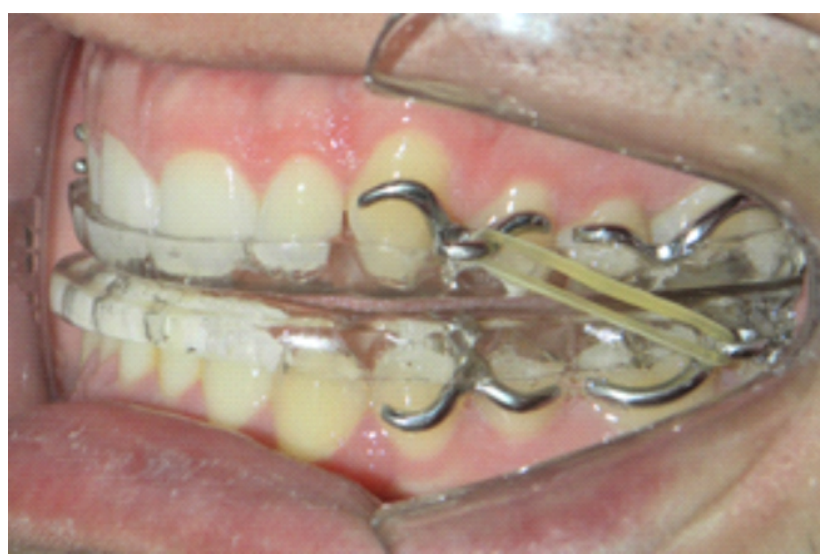

Figura 4 - Fotografias intrabucais com o AAM em posição.

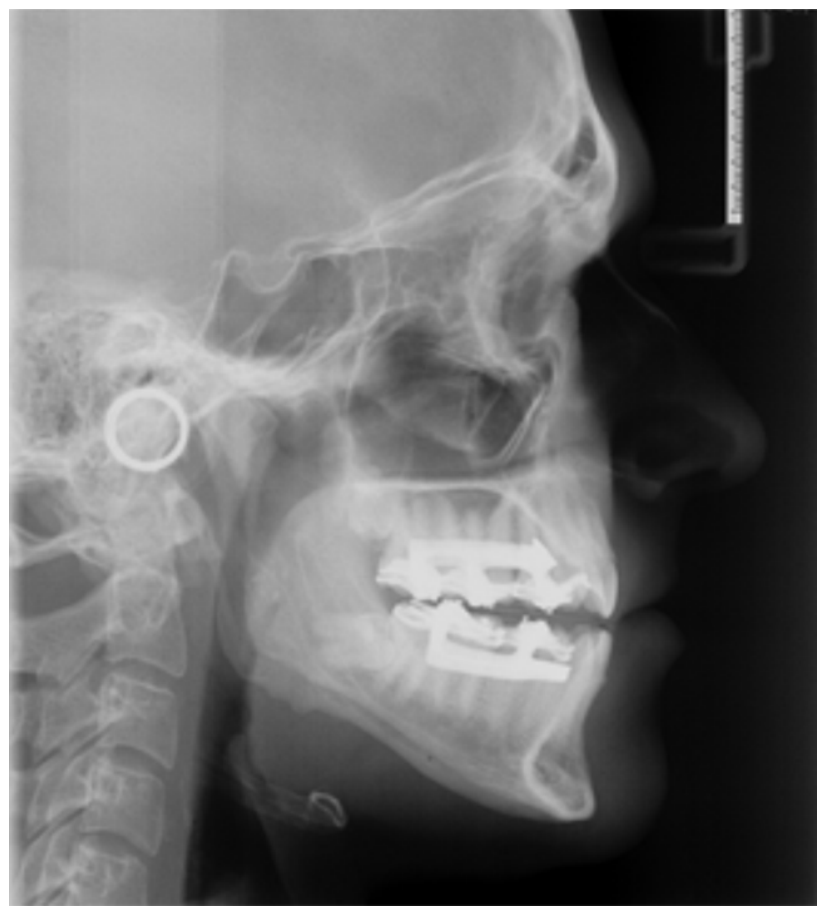

Figura 5 - Telerradiografia em norma lateral com o AAM em boca, tomada 1 ano após o início da terapia.

Tabela 1 - Apresentação dos resultados obtidos nos exames polissonográficos realizados antes e após um ano de utilização do AAM.

\begin{tabular}{|lcc|}
\hline Variável & $\begin{array}{c}\text { Valores } \\
\text { iniciais }\end{array}$ & $\begin{array}{c}\text { Valores com 1 ano de } \\
\text { terapia }\end{array}$ \\
\hline Eficiência do sono & $84.3 \%$ & $93 \%$ \\
\hline Saturação basal & $94 \%$ & $96 \%$ \\
\hline Saturação de oxihemoglobina & $88 \%$ & $90 \%$ \\
\hline IDR & 3.7 & 2.9 \\
\hline Presença de ronco & moderado & ausente \\
\hline
\end{tabular}

Após isto o paciente foi orientado a iniciar a correção de sua má oclusão. Porém, talvez por motivos particulares, o paciente não mais retornou às suas consultas agendadas, ficando ausente por 4 anos. Após este período, retornou à consulta, pois o aparelho estava um pouco desajustado, segundo suas próprias palavras. O paciente foi indagado sobre a situação de ronco neste período que esteve ausente e o mesmo relatou que não tinha mais quadro de ronco noturno e que a sensação de xerostomia matinal também havia desaparecido. Desta maneira, o aparelho foi ajustado e novas imagens foram tomadas (fotografias intrabucais, radiografia panorâmica e telerradiografia em norma lateral) (Figuras 6,7 e 8).
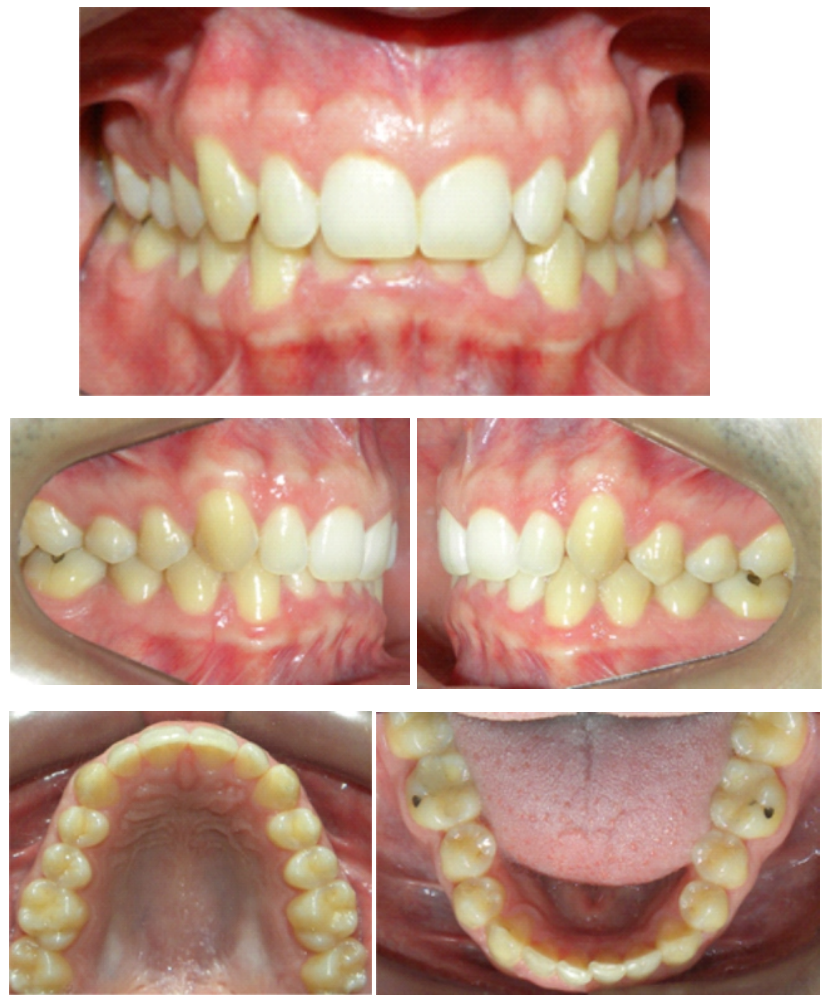

Figura 6 - Fotografias intrabucais 5 anos após o início da terapia.

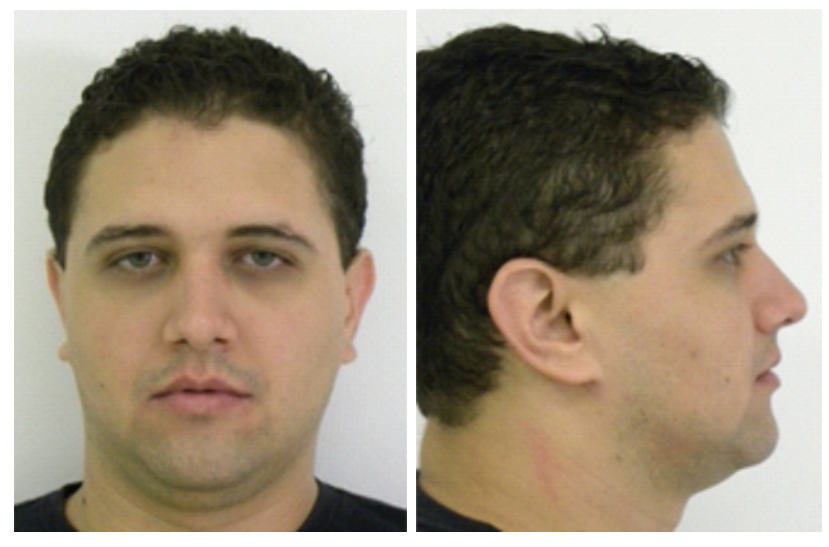




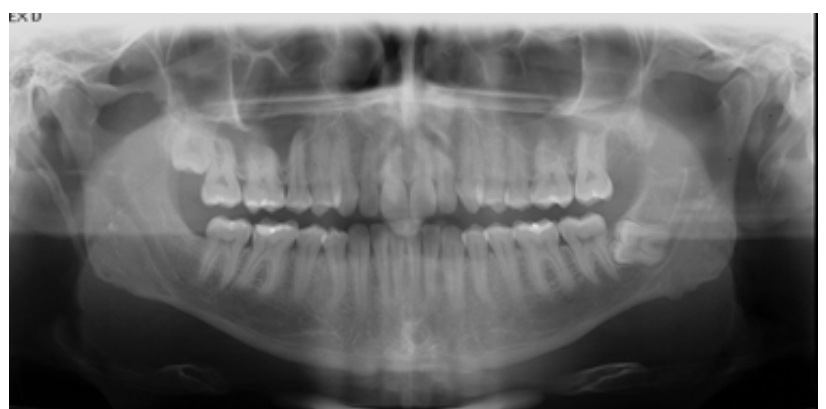

Figura 7 - Radiografia panorâmica 5 anos após o início da terapia.

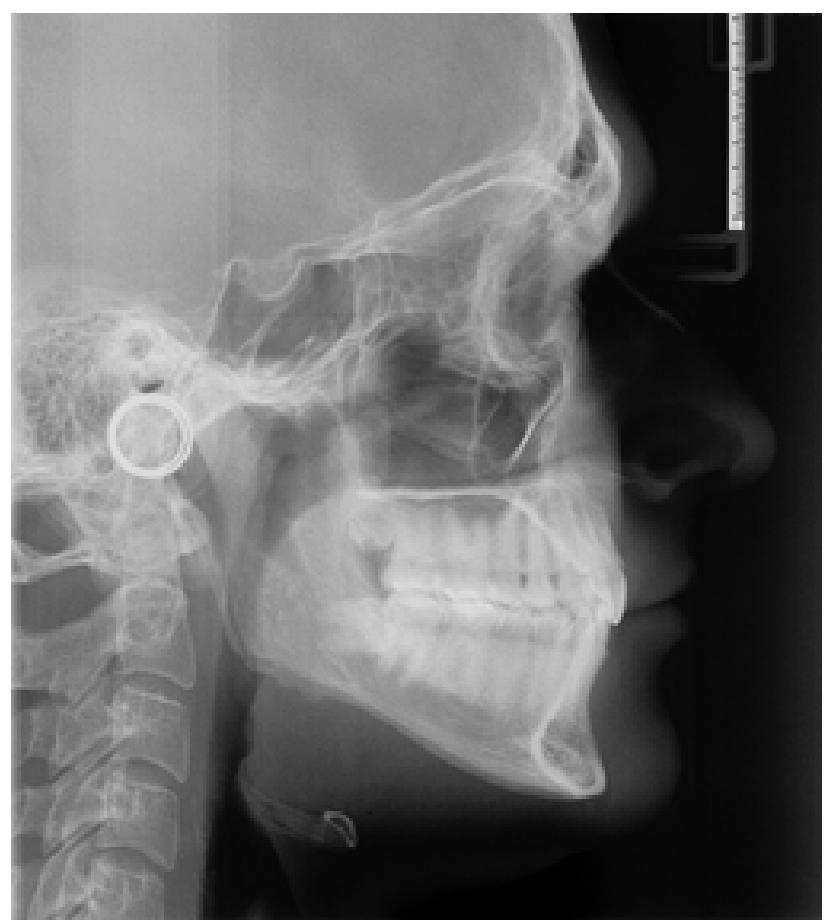

Figura 8 - Telerradiografia em norma lateral 5 anos após o início da terapia.

Com a obtenção destas imagens pôde-se realizar os traçados cefalométricos das telerradiografias inicial e 5 anos após (Figura 9). Para a realização dos traçados e também das mensurações cefalométricas, utilizou-se o programa Dolphin Imaging 11.7 (Patterson Dental Supply, Inc. USA). As alterações dentoesqueléticas promovidas ao longo de 5 anos de utilização noturna do AAM encontramse na Tabela 2.

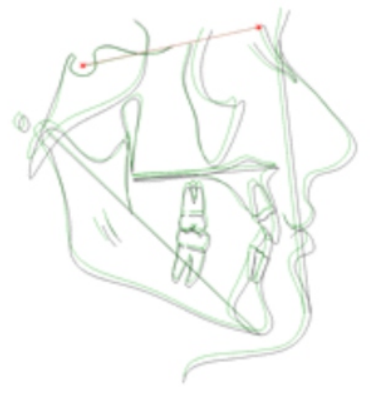

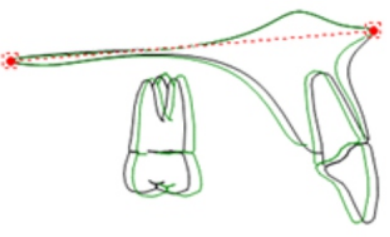

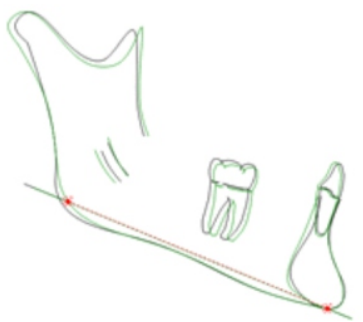

Figura 9 - Sobreposições dos traçados inicial (preto) e 5 anos após o início da terapia (verde).

Tabela 2 - Apresentação das medidas cefalométricas analisadas divididas em grupos de interpretação, com valores normativos, valores ao início do tratamento e valores após 5 anos de utilização do AAM.

\begin{tabular}{|c|c|c|c|}
\hline Grupo/ Medidas & Normativa & $\begin{array}{l}\text { Valores } \\
\text { Iniciais }\end{array}$ & $\begin{array}{c}\text { Valores } \\
\text { Finais }\end{array}$ \\
\hline \multicolumn{4}{|l|}{ Esqueléticas } \\
\hline $\operatorname{SNA}\left({ }^{0}\right)$ & 82.0 & 80.6 & 81.5 \\
\hline SNB $\left(^{\circ}\right)$ & 80.0 & 78.7 & 79.1 \\
\hline ANB $\left({ }^{\circ}\right)$ & 2.0 & 1.9 & 2.4 \\
\hline \multicolumn{4}{|l|}{ Presença de ronco } \\
\hline \multicolumn{4}{|l|}{ Padrão de Crescimento } \\
\hline SN.Gn $\left({ }^{0}\right)$ & 67.0 & 67.3 & 66.8 \\
\hline SN.GoMe $\left({ }^{0}\right)$ & 32.0 & 33.3 & 32.1 \\
\hline FMA $\left(^{0}\right)$ & 25.0 & 23.5 & 24.3 \\
\hline \multicolumn{4}{|l|}{ Incisivos e Bases Ósseas } \\
\hline 1.NA $\left(^{0}\right)$ & 22.0 & 13.3 & 12.5 \\
\hline 1-NA (mm) & 4.0 & 3.1 & 2.8 \\
\hline 1.NB $\left({ }^{\circ}\right)$ & 2.0 & 14.6 & 12.0 \\
\hline 1-NB (mm) & 4.0 & 1.8 & 1.8 \\
\hline $1.1\left(^{\circ}\right)$ & 131.0 & 150.0 & 153.2 \\
\hline IMPA $\left({ }^{\circ}\right)$ & 87.0 & 84.6 & 82.0 \\
\hline Linha I (mm) & 0.0 & 1.0 & 1.2 \\
\hline Perfil Tegumentar & & & \\
\hline
\end{tabular}

\section{DISCUSSÃO}

Há na literatura indicações de que uma SAOS severa deva ser tratada cirurgicamente, ${ }^{6,17,19}$ ou até mesmo com a utilização dos CPAPs ${ }^{9}$. A terapia com AAM apresenta sua melhor indicação para casos de SAOS leve ou moderada. Mas apesar destas diferentes indicações, tanto o tratamento com CPAPs quanto com AAMs reduzem os eventos 
obstrutivos, o cansaço diurno, o ronco, normalizam o sono, melhoram o sistema cardiovascular, a saturação de oxigênio, a qualidade de vida e a pressão sanguínea, ${ }^{8,11,20-21}$. Assim, no caso clínico apresentado neste trabalho, indicouse a utilização do AAM, pois tratava-se de um quadro de SAOS leve. Esta terapia possibilitou a protrusão mandibular conseguida pelo AAM, parecendo aumentar o diâmetro da luz do trato respiratório sem restrições. Deste modo, há uma melhora na postura da língua e consequentemente o retorno de uma deglutição normal, durante o sono, diminuindo o quadro de ronco ${ }^{2,6-7,9-15}$.

Alguns estudos indicam que os AAMs promovem diversos efeitos colaterais como disfunção temporomandibular (DTM), movimentos dentários indesejáveis, diminuição da sobressaliência (overjet), diminuição da sobremordida profunda (overbite), dor nos dentes e dor miofacia ${ }^{12,12,16-17,22}$. Contudo, muitos desses efeitos são transitórios e bem tolerados pelo paciente ${ }^{11,17}$. Outros autores afirmam que os efeitos colaterais que causam movimentações indesejáveis nos dentes só ocorrem a longo prazo ${ }^{17,20,23}$. Ao longo dos 5 anos de utilização do AAM, o paciente em questão não relatou qualquer alteração na sua articulação e tampouco de dores dentárias ou miofaciais. Pela comparação das mensurações obtidas ao início e após 5 anos de utilização do AAM, verifica-se que algumas medidas dentárias sofreram pequenas alterações, como o ângulo interincisivos, o IMPA, 1.NA e 1.NB. Porém, sem qualquer significância clínica. Também não houve alterações esqueléticas significativas, o que já era esperado, uma vez que se trata de um paciente adulto. Há também relatos na literatura pertinente que a utilização de AAM promove uma diminuição no número de contatos oclusais na região de pré-molares, devido ao avanço mandibular ser guiado pelos incisivos, permitindo uma abertura na mordida posterior $^{22}$. No caso clínico apresentado neste trabalho, o AAM foi confeccionado com uma base de acrílico recobrindo os incisivos inferiores para evitar ou minimizar este efeito colateral. Ao final de 5 anos não ocorreu esta abertura de mordida na região de pré-molares, como pode ser verificado nas imagens intrabucais finais (Figura 7).

Obviamente, os resultados aqui apresentados e discutidos relacionam-se a um paciente em particular e não devem ser tomados como normativos. Todavia, seria interessante que tais resultados pudessem ser confirmados ou não em futuros trabalhos de pesquisa, com uma amostra similar ao caso apresentado neste trabalho, ou seja, de pacientes adultos e com dentadura permanente completa.

\section{CONCLUSÃO}

Os AAMs são bem indicados para pacientes com ronco ou SAOS leve.
A utilização dos AAMs (aparelho de ITO) parece não promover alterações dentárias com relevância clínica, ao longo de 5 anos de terapia.

No caso apresentado, também não houve alterações esqueléticas significativas, a longo prazo.

\section{REFERÊNCIAS}

1. Banno K, Kryger MH. Sleep apnea: clinical investigations in humans. Sleep Med. 2007;8(4):400-26.

2. Doff MHJ, Finnema KJ, Hoekema A, Wijkstra PJ, de Bont LGM, Stegenga B. Long-term oral appliance therapy in obstructive sleep apnea syndrome: a controlled study on dental side effects. Clin Oral Invest 2013;17:475-82.

3. Bailey DR. Use of portable monitoring for sleep-disordered breathing treated with an oral appliance. Dent Clin N Am. 2012;56:445-52.

4. Merritt SL, Berger BE. Obstructive sleep apnea-hypopnea syndrome. Am J Nurs. 2004;104(7):49-52.

5. Shigemoto S, Shigeta Y, Nejima J, Ogawa T, Matsuka Y, Clark GT. Diagnosis and treatment for obstructive sleep apnea: Fundamental and clinical knowledge in obstructive sleep apnea. J Prosthodont Res. 2015;59(3):161-71.

6. Ahrens A, McGrath C, Hagg U. A systematic review of the efficacy of oral appliance design in the management of obstructive sleep apnoea. Eur J Orthod. 2011;33(3):318-24.

7. Almeida FR, Henrich N, Marra C, Lynd LD, Lowe AA, Tsuda H, et al. Patient preferences and experiences of CPAP and oral appliances for the treatment of obstructive sleep apnea: a qualitative analysis. Sleep Breath. 2013;17(2):659-66.

8. El-Solh AA, Moitheennazima B, Akinnusi ME, Churder PM, Lafornara AM. Combined oral appliance and positive airway pressure therapy for obstructive sleep apnea: a pilot study. Sleep Breath.2011;15(2):203-8.

9. Holley AB, Lettieri CJ, Shah AA. Efficacy of an adjustable oral appliance and comparison with continuous positive airway pressure for the treatment of obstructive sleep apnea syndrome. Chest. 2011;140(6):1511-6.

10. Lee CH, Mo JH, Choi IJ, Lee HJ, Seo BS, Kim DY, et al. The mandibular advancement device and patient selection in the treatment of obstructive sleep apnea. Arch Otolaryngol Head Neck Surg. 2009;135(5):439-44.

11. PliskaBT, Almeida FR. Effectiveness and outcome of oral appliance therapy. Dent Clin North Am. 2012;56(2):433-44.

12. Pliska BT, Nam H, Chen H, Lowe AA, Almeida FR. Obstructive sleep apnea and mandibular advancement splints: occlusal effects and progression of changes associated with a decade of treatment. J Clin Sleep Med. 2014;10(12):1285-91.

13. Ramar K, Dort LC, Katz SG, Lettieri CJ, Harrod CG, Thomas SM, et al. Clinical practice guideline for the treatment of obstructive sleep apnea and snoring with oral appliance therapy: an update for 2015. J Clin Sleep Med. 2015;11(7):773-827.

14. Geoghegana F, Ahrensb A, McGrathc C, Häggd U. An evaluation of 
two different mandibular advancement devices on craniofacial characteristics and upper airway dimensions of Chinese adult obstructive sleep apnea patients. Angle Orthod. 2015;85(6):962-8.

15. Dainesi EA, Schwertner A, Lima LM, Kawauchi MY. Terapia ortodôntica para pacientes com apneia obstrutiva do sono. Innov Implant J Biomater Esthet. 2014;8(1):64-9.

16. Wang X, Gong X, Yu Z, Gao X, Zhao Y. Follow-up study of dental and skeletal changes in patients with obstructive sleep apnea and hypopnea syndrome with long-term treatment with the Silensor appliance. Am J Orthod Dentofacial Orthop. 2015;147:559-65.

17. Martinez-Gomis J, Willaert E, Nogues L, Pascual M, Somoza M, Monasterio C. Five years of sleep apnea treatment with a mandibular advancement device. Side effects and technical complications. Angle Orthod. 2010;80(1):30-6.

18. Ito TR, Melo ACM, Bronzi ES. Anti-snoring appliance: a multidisciplinary focus. Rev Dental Press Ortodon Ortop Facial. 2012;5(2):48-53.

19. Almeida FR, Parker JA, Hodges JS, Lowe AA, Ferguson KA. Effect of a titration polysomnogram on treatment success with a mandibular repositioning appliance. J Clin Sleep Med. 2009;5(3):198-204.

20. Aarab G, Lobbezoo F, Heymans MW, Hamburger HL, Naeije M. Long-term follow-up of a randomized controlled trial of oral appliance therapy in obstructive sleep apnea. Respiration. 2011;82(2):162-8

21. Gauthier L, Laberge L, Beaudry M, Laforte M, Rompre PH, Lavigne GJ. Efficacy of two mandibular advancement appliances in the management of snoring and mild-moderate sleep apnea: a cross-over randomized study. Sleep Med. 2009;10(3):329-36.

22. Doff MH, Finnema KJ, Hoekema A, Wijkstra PJ, De Bont LG, Stegenga B. Long-term oral appliance therapy in obstructive sleep apnea syndrome: a controlled study on dental side effects. Clin Oral Investig. 2013;17(2):475-82.

23. Doff MH, Veldhuis SK, Hoekema A, Slater JJ, Wijkstra PJ, De Bont LG, et al. Long-term oral appliance therapy in obstructive sleep apnea syndrome: a controlled study on temporomandibular side effects. Clin Oral Investig. 2012;16(3):689-97. 